\title{
Affording inclusive dyslexia-friendly online text reading
}

\author{
Chwen Jen Chen ${ }^{1}$ Melissa Wei Yin Keong ${ }^{1}$
}

(C) Springer-Verlag Berlin Heidelberg 2016

\begin{abstract}
To date, guidelines for designing inclusive dyslexia-friendly online learning environments, which take into consideration both learners with and without dyslexia, are still scarce. As web text is one of the extensively used elements in online learning, this study aims to derive practical guidelines on this aspect by exploring the experience of learners with dyslexia and learners without dyslexia when using different online reading affordances. The study employed a within-subjects qualitative study and key patterns that emerged from the data collected via observations and interviews were interpreted based on two important aspects of learning experience, which were perceived learning and engagement. The study reveals that (1) the direct application of Printed Text on the web should be carefully considered, (2) existing web accessibility guidelines (limit to guidelines examined in this study) are appropriate and (3) the use of a Screen Reader for online reading should not be made compulsory and be available as an option instead. The comparison between the experience of learners with and without dyslexia in this study has yielded insights into affordances that are perceived positively by both groups of learners. As learners with dyslexia form a significant minority of the online learning population, the inclusive dyslexia-friendly guidelines derived from this study would better inform the future implementation of online reading affordances that acknowledge differences and similarities between online learners.
\end{abstract}

Chwen Jen Chen

cjchen@unimas.my

1 Faculty of Cognitive Sciences and Human Development, Universiti Malaysia Sarawak, 94300 Kota Samarahan, Sarawak, Malaysia
Keywords Online text reading · Dyslexia - Inclusive · Guidelines

\section{Introduction}

Dyslexia is a language-based learning disability in which individuals experience difficulty in performing languagerelated tasks such as word recognition or reading, writing, spelling, reading comprehension and sometimes speaking [82]. It is estimated that as many as $15-20 \%$ of the school population in the USA exhibit some symptoms of dyslexia [87].

The pervasive use of online learning at all levels of education nowadays calls for the crucial need to create inclusive online learning environments. Inclusive learning acknowledges differences between learners and aims to nurture an atmosphere where all learners actively and comfortably engage in their learning. As persons with dyslexia form a significant minority in the online population, their online learning needs and expectations should not be ignored. McCarthy and Swierenga [55], who have carried out a research review on dyslexia and web accessibility, report a number of web accessibility guidelines for dyslexia and other disabled users. However, many of these guidelines are meant to accommodate all visual disabilities and not specifically meant to meet the needs of persons with dyslexia [23, 55]. On the other hand, guidelines by the International Organization for Standardization [44], Nielsen [60] and the US Department of Health and Human Services [90] are among the many available web design guidelines for typical users. Little is known, however, of the effort to create inclusive dyslexiafriendly web design guidelines, particularly for online learning purposes. 